\title{
Propofol and green urine discoloration
}

\section{Lopes C.G. ${ }^{1}$, Nunes R.R. ${ }^{2}$, Cavalcante S.L.F. ${ }^{2}$, Fernandes M.B.C. ${ }^{2}$, Ribeiro K.G. ${ }^{2}$}

1Hospital Sao Carlos, Dept of Anaesthesiology, Fortaleza, Brazil, 2HGF-Hospital Geral de Fortaleza, Dept of Anaesthesiology, Fortaleza, Brazil

Background: Urine discoloration resulting from drugs, dyes, infections and ingested substances has been described in the literature. Propofol, an alkylphenol used in sedation and anesthesia, is known to induce pink, white, green and brown urine discoloration.

Case report: A 25-year old woman $(58 \mathrm{~kg}, 1,55 \mathrm{~m}$, P1) was submitted to dermolipectomy and correction of incisional hernia. Epidural anesthesia was administered at T12-L1 using levobupivacaine $(0.5 \%)$ with epinephrine $(1: 200.000)$ and sufentanil $(20 \mu \mathrm{g})$. Sedation was achieved and maintained with a continuous infusion of propofol (1-2 $\mu \mathrm{g} \cdot \mathrm{mL}-1$ at the effector site). By the end of the procedure, which lasted 3.5 hours, the bladder contained 300 $\mathrm{mL}$ green urine. No further investigations were conducted and the discoloration resolved spontaneously within 3 hours.

Discussion: Green urine discoloration is usually caused by the ingestion of substances such as amitriptyline, cimetidine, promethazine, indometacin, triamterene, methylene blue and dyes found in chewing gum and mouthwash. Discoloration may also be caused by pathologies, such as urinary Pseudomonas infection, obstructive jaundice, urinary tract fistula and Hartnup disease. Propofol is metabolized mainly by the liver, but also to some extent by the kidneys, lungs and bowels.
The metabolites-water-soluble glucuronides and sulfate conjugates excreted by the kidneys-are believed to confer a green color to the urine but are biologically inactive and do not affect nor reflect kidney function. Urine alkalinization tends to increase the production of metabolites. Though rare, discoloration is most likely to occur after long infusions, but has been observed after short infusions or even after a single dose. The symptom resolves as early as 2-3 hours after infusion is stopped

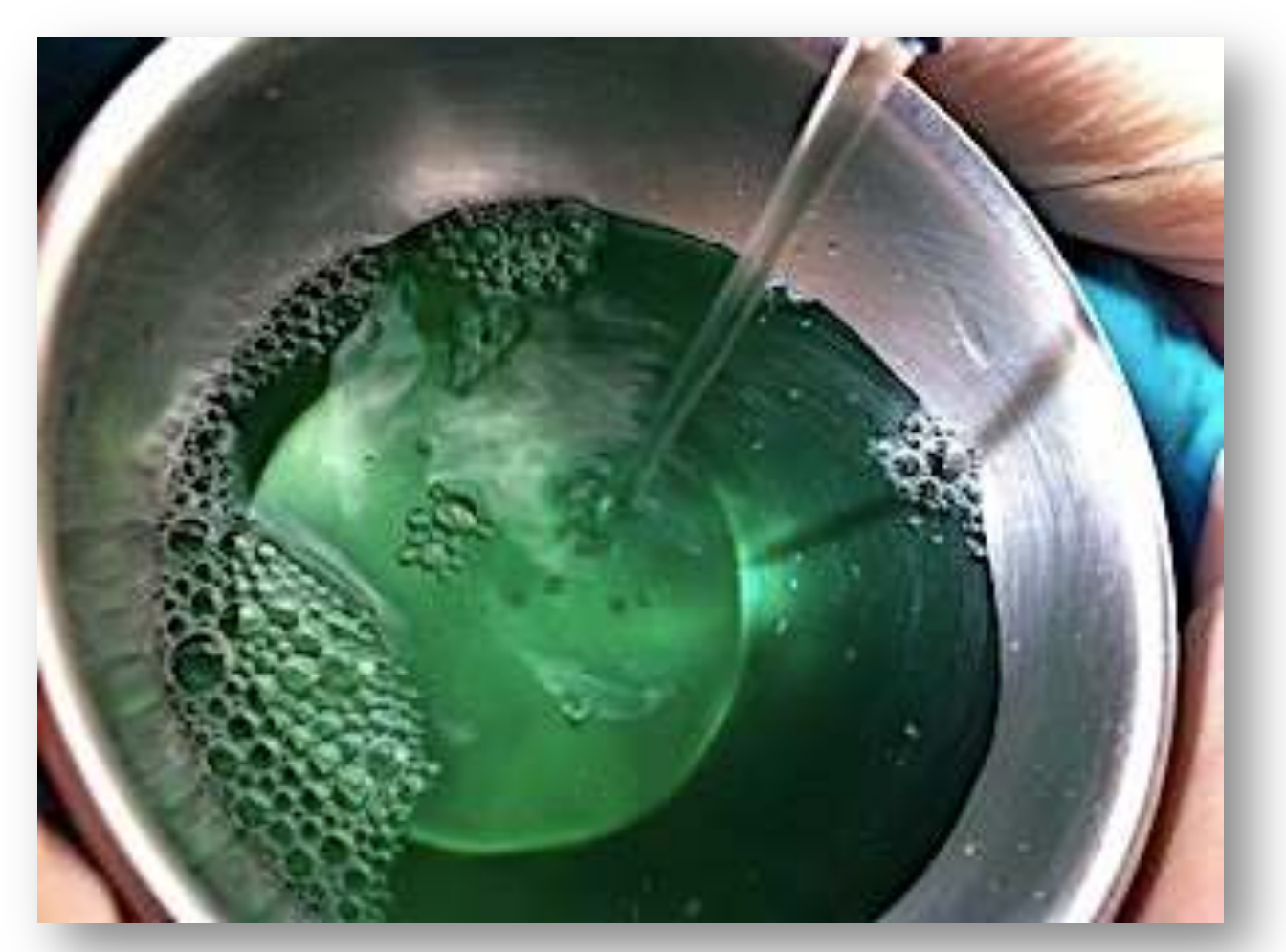

Green urine

References: 1.Blakey SA, Hixson-Wallace JA. Clinical significance of rare and benign side effects: propofol and green urine.Pharmacotherapy 2000;20:1120-1122 